INPLASY

PROTOCOL

To cite: Zhang et al. Efficacy and safety of Shenling Atractylodes Powder in the treatment of ulcerative colitis: a systematic review and Meta analysis. Inplasy protocol 202110084. doi:

10.37766/inplasy2021.1.0084

Received: 21 January 2021

Published: 21 January 2021

Corresponding author:

Quanhui Zhang

36791584@qq.com

Author Affiliation:

Affiliated Hospital of Jiangxi University of Traditional

Chinese Medicine

Support: None.

Review Stage at time of this submission: The review has not yet started.

Conflicts of interest: None.

\section{Efficacy and safety of Shenling Atractylodes Powder in the treatment of ulcerative colitis: a systematic review and Meta analysis}

Zhang, QH1; Deng, YW2; Wang, JL33 Haung, FH4; Zhou, YD5; Jia, MY6; Yi, HR7.

Review question / Objective: The purpose of this study was to explore the clinical efficacy and safety of shenling baizhu powder in the treatment of ulcerative colitis through systematic evaluation and Meta analysis.

Condition being studied: Ulcerative colitis (UC) is a kind of chronic non-specific ulcerative colitis, which is characterized by repeated abdominal pain, diarrhea and mucus purulent stool. The disease is more recurrent, easy to delay, and canceration, seriously affect the quality of life, increase the economic burden of patients and society, treatment is more difficult, the World Health Organization as one of the modern refractory diseases.Shenling Atractylodes Powder in the treatment of ulcerative colitis showed a strong advantage, the effect is accurate. therefore, this paper will systematically evaluate and Meta analyze the efficacy and safety of heatsensitive moxibustion in the treatment of ulcerative colitis.

INPLASY registration number: This protocol was registered with the International Platform of Registered Systematic Review and Meta-Analysis Protocols (INPLASY) on 21 January 2021 and was last updated on 21 January 2021 (registration number INPLASY202110084).

\section{INTRODUCTION}

Review question / Objective: The purpose of this study was to explore the clinical efficacy and safety of shenling baizhu powder in the treatment of ulcerative colitis through systematic evaluation and Meta analysis.

Condition being studied: Ulcerative colitis (UC) is a kind of chronic non-specific ulcerative colitis, which is characterized by repeated abdominal pain, diarrhea and 
mucus purulent stool. The disease is more recurrent, easy to delay, and canceration, seriously affect the quality of life, increase the economic burden of patients and society, treatment is more difficult, the World Health Organization as one of the modern refractory diseases.Shenling Atractylodes Powder in the treatment of ulcerative colitis showed a strong advantage, the effect is accurate. therefore, this paper will systematically evaluate and Meta analyze the efficacy and safety of heat-sensitive moxibustion in the treatment of ulcerative colitis.

\section{METHODS}

Participant or population: Have clear and recognized diagnostic criteria and efficacy criteria, and all patients were diagnosed UC, there are no restrictions on the sex, age, occupation, course of disease, source of cases.

Intervention: Oral Shenling Atractylodes Powder plus or minus prescription therapy, or based on oral Shenling Atractylodes Powder plus or minus prescription will also be included.

Comparator: The control group will receive one of the following treatment methods: conventional pharmacological therapy, no treatment, and placebo.

Study designs to be included: The clinical randomized controlled trial (RCTs) included Shenling Atractylodes Powder and UC, the publication state is unlimited, the language is Chinese or English.

Eligibility criteria: Reported in chinese and Engish,and meet the "PICOS", will be considered for inclusion in this overview.

Information sources: PubMed, Embase, Web of Science, Cochrane Library, the China National Knowledge Infrastructure, Chinese Science and Technology Periodical Database, Wanfang Database, and Chinese Biomedical Literature Database.

Main outcome(s): Colonic mucosal symptom score, total effective rate, clinical symptom score, recurrence rate Mayo enteroscopy grading.

Additional outcome(s): IL-6、IL-9、TNF-a、 IL-4、IL-10 the level of inflammatory related factors and the total incidence of adverse reactions.

Data management: The two researchers independently read the title and abstract of the literature we obtained, read the full text of the trials that might meet the inclusion criteria to determine whether the inclusion criteria were truly met, and discussed the conflicting literatures or let the third researcher decide whether to include them. Two researchers independently extracted data from the included studies, including study design, intervention measures and methods, measurement indicators, results, methodological contents such as hidden grouping and blind method, etc., and a third evaluator checked the consistency of the data. If the required information is incomplete, we will contact the original author for the required data.

Quality assessment / Risk of bias analysis: Two evaluators independently select the literature according to the inclusion and exclusion criteria and cross-check. In case of disagreement, a third evaluator will assist in the decision. The extracted data included the first author, year of publication, number of patients, age, gender, intervention measures, outcome indicators, etc. The Jadad scale to evaluate quality into literature, including: random sequence (right 2 points, 1 points not clear, inappropriate 0 ), distribution, hidden (right 2 points, 1 points not clear, inappropriate 0 ), blinded (right 2 points, 1 points not clear, inappropriate 0 ), lost to follow-up and exit (describe 1 points, not describe 0 ); $0-3$ is classified as low quality and 4-7 as high quality.

Strategy of data synthesis: Meta analysis will be performed using Rev Man5.3.0 software. The odds ratio (OR) and its $95 \%$ Confidence Interval ( $\mathrm{Cl}$ ) will be used as the counting data, while the weighted mean 
difference (WMD) and its $95 \% \mathrm{Cl}$ will be used as the measurement data.

Subgroup analysis: The heterogeneity test will be carried out first among all studies, 12 test will be used. When $P>0.1$ and $I 2<$ $50 \%$, the fixed effect model will be used; otherwise, the random effect model will be used. When the clinical heterogeneity between the two studies is large, only descriptive analysis will be performed.

Sensibility analysis: The purpose of sensitivity analysis is to determine the sources and confounding factors of heterogeneity. If the trial data is sufficient, low or high quality studies will be excluded one by one for sensitivity analysis.

Language: No limitation of language.

Country(ies) involved: China.

Keywords: Shenling Atractylodes Powder plus or minus; ulcerative colitis; systematic review and Meta analysis.

Contributions of each author:

Author 1 - Quanhui Zhang - The author drafted the manuscript.

Email: 36791584@qq.com

Author 2 - Yongwen Deng - The author provided statistical expertise.

Email: dywen@163.com

Author 3 - Jinlong Wang - The author contributed to the development of the selection criteria, and the risk of bias assessment strategy.

Email: 1349556348@qq.com

Author 4 - Feihong Huang - The author read, provided feedback and approved the final manuscript.

Email: 1186404149@qq.com

Author 5 - Yiduo Zhou - The author provided statistical expertise.

Email: 1686193469@qq.com

Author 6 - Mingyan Jia - The author provided statistical expertise.

Email: jiamingyany@163.com

Author 7 - Haoran Yi - The author contributed to the development of the selection criteria.

Email: 1515418321@qq.com 